Original title / titulo original La "Piedra del Sol" y la relectura de los mitos cosmogónicos

\title{
Author(s)/ autor(es):
}

\section{Elżbieta Siarkiewicz}

Published originally as/ Publicado originalmente en: Estudios Latinoamericanos, 19 (1999), pp. 87-100.

DOI: https://doi.org/10.36447/Estudios1999.v19.art5

Estudios Latinoamericanos is a journal published by the Polish Society for Latin American Studies (Polskie Towarzystwo Studiów Latynoamerykanistycznych).

The Polish Society for Latin American Studies is scholarly organization established to facilitate research on Latin America and to encourage and promote scientific and cultural cooperation between Poland and Latin America.

Estudios Latinoamericanos, revista publicada por la Sociedad Polaca de Estudios Latinoamericanos (Polskie Towarzystwo Studiów Latynoamerykanistycznych).

Sociedad Polaca de Estudios Latinoamericanos es una asociación científica fundada con el fin de desarrollar investigaciones científicas sobre América Latina y participar en la cooperación científica y cultural entre las sociedades de Polonia y América 


\title{
La "Piedra del Sol" y la relectura de los mitos cosmogónicos
}

\author{
Elżbieta Siarkiewicz \\ Cátedra de Estudios Ibéricos \\ Universidad de Varsovia
}

El monolito conocido como la "Piedra del Sol" o "Calendario Azteca" (fig. 1) es uno de los monumentos precolombinos más conocidos en el mundo. Hasta en Varsovia podemos comprar sus réplicas miniaturizadas de bronce, en tiendas especializadas en la venta de objetos esotéricos.

El monumento fue descubierto en el año 1790, al ampliar y nivelar la Plaza Mayor de la Ciudad de México. Durante varios años el monumento permaneció adosado al costado de una de las torres de la Catedral, hasta que en 1885 fue trasladado al Museo Nacional de México. Hoy domina en la Sala Mexica del Museo Nacional de Antropología e Historia, donde atrae no sólo a los turistas. Desde su descubrimiento se ha transformado en una fuente de inspiración de hombres de letras y de artistas, y sigue siendo, a pesar del tiempo transcurrido, un reto para los investigadores de las culturas prehispánicas en las Américas.

La "Piedra del Sol" está hecha de basalto. La parte cubierta de relieves tiene $3,5 \mathrm{~m}$ de diámetro ( $20 \mathrm{~cm}$ en el canto). Probablemente su posición original era horizontal y estaba cubierta de un techo provisiorio (hoy el monumento se encuentra expuesto verticalmente). El ornamento consiste en una serie de ruedas concéntricas, que desde la banda exterior hacia la interior se compone de:

1. El canto - símbolos del planeta Venus separados por cuchillos de obsidiana (fig. 2).

2. Dos Xiuhcoatles - Ígneos < dragones $>$ celestes, con la fecha 13-Acatl entre las colas (fig. 3).

3. 8 símbolos de rayos de luz, 40 quincunces y 8 colgajos de jadeita (fig. 4).

4. 20 glifos que representan signos diurnos (fig. 5). 
5. La rueda interior con el nombre del día 4-0llin (fig. 6) (ampliado, en el cual se encuentran inscritos, en cuadretes, otros cuatro nombres del día: 4-0celotl, 4-Ehecatl, 4-Quiahuitl, y 4-Atl (fig. 7); y en el círculo central, una cara humana, de frente). Aparecen además tres nombres del día adicionales: 1-Tecpatl (arriba, junto al glifo onomástico de Motecuhzoma), 1-Quiahuitl y 7-0zomatli (abajo).

Los mencionados símbolos que recubren su superficie, han permitido que el monolito fuera objeto de múltiples interpretaciones, y larga es la lista de los investigadores quienes dedicaron parte de sus publicaciones a este tema. Fue Herman Beyer el que realizó, en 1921, el análisis más municioso de todos los elementos iconográficos que figuran en el monumento. Según este autor ${ }^{1}$, el monolito fue dedicado al Sol y estaba vinculado con los ritos solares. Conmemoraba los tiempos míticos durante los cuales tuvo lugar la rotación de las épocas o soles cosmogónicos. Esta interpretación general es aceptada por la mayoría de los investigadores contemporáneos, las diferencias se visualizan a nivel de algunos detalles, p.ej., durante los últimos años la discusión se centra en torno al mensaje simbólico de la cara humana que figura en la parte central. ${ }^{2}$

Quisiera demostrar que fuera del Sol, existen premisas que permiten vincular la información codificada en el monumento con otros cuerpos celestes, en este caso, con la Luna y con Venus. Aunque todas las interpretaciones se refieren al tiempo mítico quisiera ampliar el enfoque e incorporar el tiempo presente que también se encuentra en el monumento. Considero que los cinco nombres del día que llevan el numeral 4 constituyen la llave para comprender esta cuestión.

Según M. Graulich, los mitos vinculados con los orígenes del Sol y de la Luna, en sus diversas versiones que provienen de diferentes áreas de Mesoamérica, surgen de una antigua fuente común ${ }^{3}$. La mayoría de las fuentes escritas afirma que el mundo pasó por una serie de épocas, llamadas también eras o soles, consistentes en unas destruc-

1 Herman BEYER, El llamado <Calendario Azteca > : descriptión e interpretación del cuaubxicalli de la <Casa de las Águilas>, Verband Deutscher Reichsangehoriger, México, 1921.

2 Para C. Klein, representa al Sol en el inframundo, al Sol Nocturno; para D. Heyden, C. Navarrete y R.F. Townsend a la deidad telúrica, Tlaltecuhtli: según M. Graulich, es el sol del mediodía. H.B. NICHOLSON reconsidera todas estas opciones, y confirma la vieja teoría de que la cara pertenece a Tonatiuh - la deidad solar [The problem of the identification of the central image of the <Aztec Calendar Stone>, en Current Topics in Aztec Studies, A. Cordy-Collins \& D. Sharon, Eds, San Diego Museum Papers 30, 1993, pp. 3-15]. Recientemente, S. MILBRATH opta por interpretar a la figura central como al sol diurno eclipsado [Eclipse imagery in Mexica sculpiure of Central Mexico, $\mathrm{PO}$ nencia presentada durante el simposio Inspiration of Astronomical Phenomena, Roma, 1994]. 
clones y construcciones cíclicas. Los agentes de estos fines del mundo eran los dioses y las fechas de las destrucciones son las que figuran inscritas en el signo central del monumento, ollin. Las fuentes no están de acuerdo en cuanto al nombre del día del primero o del último cataclismo, ni cuanto duró cada época (se menciona dos ciclos de 676, y otros dos de 364 y 312 años), pero concuerdan con la idea principal. El orden de la secuencia de fechas en la Leyenda de los Soles y en Historia de los Mexicanos por sus Pinturas coincide con las que aparecen en la "Piedra del Sol" ${ }^{4}$, por eso vamos a referirnos, sobre todo, a estos dos manuscritos.

Si comenzamos el análisis por el cuadrete ubicado arriba, a la derecha, donde figura el signo 4-0celotl, según las mencionadas fuentes, el mundo construído por Tezcatlipoca quedó destruido en ese día por Quetzalcóatl, y los gigantes que habitaban la tierra quedaron devorados por jaguares. Quetzalcóatl reconstruyó el mundo, pero el día 4-Ehecatl, Tezcatlipoca envió un viento tan fuerte que arrasó todo, y los seres humanos se transformaron en simios. La siguiente época se encontraba bajo la tutela de Tlaloc, pero Quetzalcóatl mandó una lluvia de fuego que quemó todo, el día 4-Quiahuitl, y los seres humanos se transformaron en aves. La cuarta época era la de la deidad Chalchiuhtlicue, pero el día 4-Atl comenzó a llover, la tierra se inundó, y el cielo cayó sobre ella, transformándose los seres humanos en animales acuáticos.

Lo que es característico, es que las fuentes no siempre mencionan a la deidad destructora, puesto que los nombres de los días, aparte de designar la fecha de la destrucción, simbolizan a los dioses protectores de cada época, por lo que pueden simbolizar también el primer, y no sólo el último día de cada una de ellas. Según la Historia de los Mexicanos por sus Pinturas, después de la última catástrofe, Quetzalcóatl junto con Tezcatlipoca y con la ayuda de cuatro seres humanos y dos árboles, lograron levantar el cielo de la tierra ${ }^{5}$. Esto tuvo lugar el año 1-Tochtli, fecha que se repite en otros documentos. De esta manera, el mundo quedó reconstruído una vez más. Se creó el fuego el año siguiente, 2-Acatl, pero no existía la luz del Sol, reinaba la Noche.

4 Michel GRAULICH, Los mitos mexicanos y maya quicbés de la creación del Sol, en Anales de Antropología, vol. XXIV, 1987, pp. 289-325.

4 Sobre el análisis concerniente a este problema ver Ryszard TOMICKI, Estructura de las Épocas del mundo en la mitología Nabuatl: El problema de la llamada versión canónica de Tenochtitlan, en Ethnologia Polona, vol. 11, 1985, pp. 45-73.

5 Historia de los mexicanos por sus pinturas, en Teogonía e Historia de los Mexicanos, A.M. Garibay K. editor, Porrúa, México, 1979, p. 32. 
Después de 25 años, el año 13-Acatl (fecha que aparece entre las colas de los Xiuhcoatles en nuestro monumento), los dioses se reunieron en Teotihuacán para crear el nuevo Sol. La creación consistiría en la transformación de uno de ellos, por medio del autosacrificio, en el Sol. Pero el autosacrificio, esta vez, requería la autoinmolación en el teotexcalli (horno divino). Existen varias versiones de esta parte del mito, pero todas tienen el mismo esquema.

Según La Leyenda de los Soles ${ }^{6}$, los dioses Tonacatecuhtli y Xiuhtecuhtli escogieron a Nanáhuatl, al pobre dios buboso, como candidato para transformarse en el Sol, en cambio, Tlalocantecuhtli y Napatecuhtli designaron a Nahuitecpatl (4-Tecpatl), o a Teccistecatl según otra versión, para que se transformara en la Luna. Después de cuatro días de penitencia ritual, Nanáhuatl saltó al fuego y se transformó en el Sol; Nahuitecpatl saltó en la ceniza y se transformó en la Luna ${ }^{7}$. Cuando el Sol apareció en el firmamento no quiso moverse durante cuatro días. Requería el sacrificio de las deidades reunidas en Teotihuacán. Uno de los dioses, Tlahuizcalpantecuhtli (Señor del Alba - Venus), enfurecido, le disparó cuatro flechas, pero no logró el efecto esperado. A su vez, fue el Sol quien disparó sus flechas contra Tlahuizcalpantecuhtli y le cubrió la cara con los nueve cie$l o s^{8}$. A continuación, el texto habla sobre el sacrificio de los dioses que tomaban parte en el rito, y nos dice también que la Luna que apareció en el firmamento después del Sol, fue arremetida por Papáztac quien le quebró una taza de figura de conejo en la cara" Según la Leyenda de los Soles, la Luna fue encontrada luego por los duendes, en una encrucijada.

Se asume que la fecha 4-0llin, presente en el monumento, es la fecha del principio del Sol actual, es decir de la quinta época, y de que éste va a ser el nombre del día de su

6 Leyenda de los Soles, en Códice Chimalpopoca, trad. P. Feliciano Velázquez, UNAM, México, 1975, pp. 121-122.

7 Según la Historia de los mexicanos por sus pinturas, Quetzalcóatl echa a su hijo en la hoguera para que se transforme en el Sol, mientras que Tlaloc echa al suyo en la cenizas para transformarlo en la Luna. Bernardino de Sahagún, en cambio, recogió otra versión del mito, según la cual el designado a transformarse en el Sol fue Tecuciztecatl, sólo que le faltó valor para echarse primero en la hoguera [Bernardino de SAHAGÚN, Florentine Codex, General History of the Things of New Spain, Ed. and trans. by Arthur J.0. Anderson and Charles E. Dibble, School of American Research and University of Utah, Santa Fe, New Mexico, 1953, LVII: 4-7].

$8 \quad$ Leyenda de los Soles, op. cit., p. 122.

9 En la versión de Sahagún, uno de los dioses le echó un conejo en la cara, por brillar la Luna así como el Sol, con lo que le quitaba el brillo. 
destrucción. El agente de la destrucción será un terremoto, pues ese sentido, aparte de movimiento, lleva la palabra Ollin. Esta interpretación de las cinco fechas calendáricas, conmemorativas del tiempo cosmológico, es la generalmente aceptada. Pero estas mismas fechas podían haber cumplido otra función en la época actual - la época de los Mexicas.

En el monumento figura el glifo 1-Tecpatl, el día del nacimiento de la deidad tribal Huitzilopochtli, pero también la fecha de la partida de los mexicas del mítico Aztlan, el principio del cómputo del tiempo histórico. En frente del glifo 1-Tecpatl aparece el glifo de la corona real y la vírgula, el símbolo de la palabra - poder y que puede ser interpretado como el glifo onomástico de Moctecuhzoma II $^{10}$. Es decir, en este ejemplo vemos que los dos glifos hacen referencia al tiempo mítico, al punto de partida de su tiempo histórico y, al mismo tiempo, a la época contemporánea, la época del imperio Mexica ${ }^{11}$.

Si asumimos que el día 4-0llin es el principio del movimiento del Sol, lo es también para la Luna, pues ésta también es una de los protagonistas del mito. Lo mismo concierne al planeta Venus, tanto más que sus glifos se encuentran en el canto del monumento. Su nombre, Tlahuizcalpantecuhtli (Señor de la Casa del Alba), y el hecho de cubrirle el Sol con los nueve cielos, pueden designar el momento de la conjunción inferior del planeta, cuando éste se torna invisible para el observador.

Aceptando, entonces, que 4-0llin es el punto de partida del cómputo de los tres cuerpos celestes, veremos que los nombres del día que aparecen en la rueda central pueden designar fechas exactas de años del trópico, meses sinódicos de la luna y revoluciones sinódicas de Venus. Para demostrarlo vamíos a tratar los glifos calendáricos que aparecen en la rueda central de la "Piedra del Sol" de la misma manera que a las inscripciones que aparecen en los tonalamatles ${ }^{12}$. Es decir, vamos a sumar $\mathbf{n} \times 260$ días entre los glifos, lo que no altera de manera alguna la inscripción misma.

En lo que concierne al cómputo solar, el mínimo múltiplo del tonalpohualli que da como resultado un definido número de años del trópico (AT) es: 59 × $260 \mathrm{~d}=15340 \mathrm{~d}$

$\overline{10}$ E. UMBERGER, A reconsideration of some bieroglypbs in the Mexica Calendar Stone, en Smoke and Mist: Mesoamerican Studies in Memory of Thelma Sullivan, J.K. Josserand \& K. Dakin, Eds, BAR International Series 402, 1988, pp. 345-388.

11 Según la interpretación de R.F. TOWNSEND [State and Cosmos in the Art of Tenochtitlan, Dumbarton Oaks, Washington 1979], la fecha 13-Acatl en el discutido monumento se refiere tanto al tiempo mitológico como al tiempo presente, al año 1427 de la intronización de Izcóatl y el inicio del poderío mexica.

12 E. SIARKIEWICZ, El Tiempo en el tonalamatl, Dialog, Warszawa, 1995. 
$=42$ AT $(-4,146 \mathrm{~h})$. Esto significa que cada 42 años del trópico se repetirá el nombre del día que inició el cómputo.

El cómputo basado en $260 \mathrm{~d}$ x $59=42 \mathrm{AT}$, puede ser reducido a intervalos menores. la "Piedra del Sol" ofrece las siguientes posibilidades:

$$
\begin{aligned}
& 0.5 \mathrm{AT}=182 \text { días } \\
& 10 \mathrm{AT}=3653 \mathrm{~d}=260 \mathrm{~d} \times 14+13 \mathrm{~d} \\
& 10.5 \mathrm{AT}=3835 \mathrm{~d}=260 \mathrm{~d} \times 14+195 \mathrm{~d} \\
& 11 \mathrm{AT}=4017 \mathrm{~d}=260 \mathrm{~d} \times 15+117 \mathrm{~d} \\
& 21 \mathrm{AT}=7670 \mathrm{~d}=260 \mathrm{~d} \times 29+130 \mathrm{~d} \\
& 31 \mathrm{AT}=11323 \mathrm{~d}=260 \mathrm{~d} \times 43+143 \mathrm{~d} \\
& 31,5 \mathrm{AT}=11505 \mathrm{~d}=260 \mathrm{~d} \times 44+65 \mathrm{~d} \\
& 42 \mathrm{AT}=15340 \mathrm{~d}=260 \mathrm{~d} \times 59
\end{aligned}
$$

$$
\begin{aligned}
& \text { (4-ollin }=>4 \text {-quiahuit) } \\
& \text { (4-atl }=>4 \text {-ehecatl) } \\
& \text { (4-quiahuill }=>4 \text {-ocelot }=>4 \text {-atl 4-ehecatl }=>4 \text {-ollin) } \\
& \text { (4-ollin }=>4 \text {-ocelotl; 4-ehecatl }=>4 \text {-quiahuitl) } \\
& \text { (4-quiahuitl }=>4 \text {-atl } \Rightarrow>4 \text {-quiahuitl...) } \\
& \text { (4-ocelotl }=>4 \text {-ollin; 4-quiahuitl }=>4 \text {-ehecatl) } \\
& \text { (4-at }=>4 \text {-ocelot }=>4 \text {-quiahuitl; } 4 \text {-ollin }=>4 \text {-ehecat) } \\
& \text { (cada signo) }
\end{aligned}
$$

A base de estos datos pueden generarse series más largas. La tabla I presenta las posibilidades del cómputo (* la primera enmienda ).

TABLA I: Número de años solares

\begin{tabular}{lccccccccc}
\hline 4-ollin & 0 & 42 & 84 & 126 & 168 & 210 & $252 *$ & 1255 & 1507 \\
4-quiahuitl & 0,5 & 42,5 & 84,5 & 126,5 & 168,5 & 210,5 & $\ldots$ & 1255,5 & 1507,5 \\
4-ocelotl & 11 & 53 & 95 & 137 & 179 & 221 & $\ldots$ & 1266 & 1518 \\
4-atl & 21,5 & 63,5 & 105,5 & 147,5 & 189,5 & 231,5 & 502 & 1276,5 & 1528,5 \\
4-ehecatl & 31,5 & 73,5 & 115,5 & 157,7 & 199,5 & 241,5 & 512 & 1286,5 & 1538,5 \\
\hline
\end{tabular}

Esta forma de cómputo permite incorporar el glifo 1-Quiahuitl cuyo significado (junto con el 7-0zomatli) no ha sido todavía satisfactoriamente interpretado. Si el cálculo principia en 4-0llin, entonces 1-Quiahuitl puede designar 8 años del trópico (2922 días $=584 \mathrm{~d} \times 5+2 \mathrm{~d}=260 \mathrm{~d} \times 11+62 \mathrm{~d})$.

1-Tecpatl también puede ser interpretado como fecha solar, y designaria 19.5 ÁT (7121días $=260 \mathrm{~d} \times 27+101 \mathrm{~d})$ después de 4-01lin (11.5 AT desde la fecha 1-Quiahuitl). El nombre del día 1-Tecpatl, como fecha solar, está vinculado numéricamente con 1-Tochtli (1-Tecpatl $+7670 \mathrm{~d}(21 \mathrm{AT})=>1$-Tochtli $+7670 \mathrm{~d}$ $=>1$-Tecpatl...). Ambas podían funcionar como fechas del medio año solar 0 tal vez como medios soles ${ }^{13}$, cada 21 años del trópico.

13 El análisis del concepto de medio sol iniciado por M. Graulich (nota 3) merece su continuación. Por ser un tema separado no será desarollado en este lugar. 
La Leyenda de los Soles proporciona otros dos nombres más para el Sol: 6-Xochitl y 6-Ehecatl y encajan muy bien en esta forma de cómputo solar: 6-Xochitl sería la fecha para el décimo segundo año del trópico $(2922 \mathrm{~d}+1461 \mathrm{~d}=4383 \mathrm{~d}=260 \mathrm{~d} \times 15+$ 220d); 6-Ehecatl situado 182 días después, marcaría la mitad del siguiente año solar. Los nombres del Sol de la Leyenda de los Soles denotan entonces: año cero, año décimo segundo, y décimo segundo y medio, y podían ser repetidos cada 59 tonalpohuallis $(59 \mathrm{x}$ $260 \mathrm{~d}=15340$ días $=42$ años del trópico). Armoniosamente suplementan la lista de los nombres del Sol que ofrece nuestro monumento, nombres que, como vemos, diferencian ciclos solares.

Como es bien sabido, los mesomericanos basaban el cómputo de Venus en la medida de 584 días. El mínimo múltiplo del tonalpohualli que ofrece la aproximación más exacta de las revoluciones sinódicas del planeta (RV), expresado en números de días enteros es - según el Códice de Dresde -137 tonalpohuallis $=61 \mathrm{RV}(260 \mathrm{~d} \times 137=$ $35620 \mathrm{~d}$. Este número de días conlleva el error de +21 h por ciclo y podía ser enmendado en cuentas más altas, cuando el error rebasaba 4 días, descontando 9 tonalpohuallis ( $260 \mathrm{~d} \times 9=2340 \mathrm{~d}=585 \mathrm{~d} \times 4=584 \mathrm{~d} \times 4+4$ días). El óptimo múltiplo al que se llega usando este método es de 676 tonalpohuallis $=301 \mathrm{RV}(260 \mathrm{~d}$ × $676=175760 \mathrm{~d})$, número de días que no necesita enmienda gracias a un error mínimo que conlleva ( 1,8 h en 481 años). Es interesante observar que 676 es el número que se repite en la cantidad de años que duraron dos de las épocas pasadas, y es el resultado de la suma de las dos épocas restantes $(364+312=676)$. Además de las medias mencionadas, hay que incluir la de 7007 días $=12 \mathrm{RV}\left(260 \mathrm{~d} \times 29-13 \mathrm{~d}^{14}\right)$, ya que parece ser ésta la que tiene marcada influencia en la codificación de las fechas venusianas en la "Piedra del Sol".

Nuestro monumento ofrece las posibles fechas de ciclos venusinos más cortos:

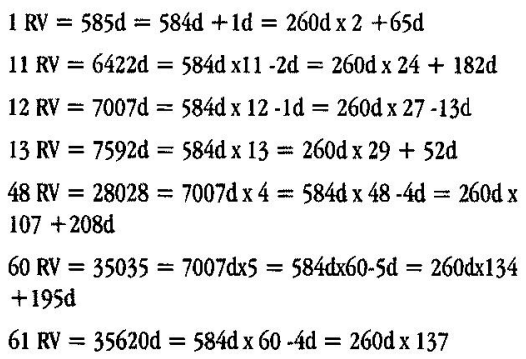

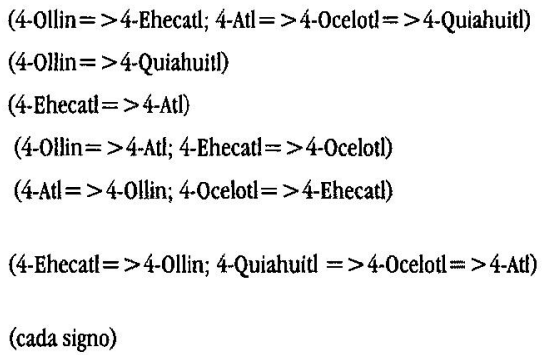

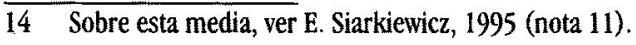




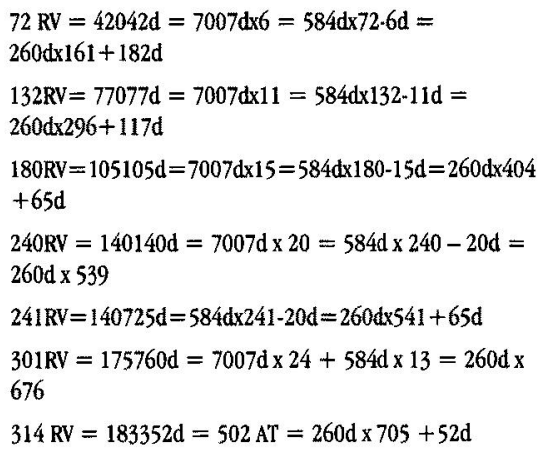

En la tabla II ofrecemos las fechas y el número de los ciclos venusinos que podrían estar codificados en la "Piedra del Sol", Vamos a basarnos en el sistema usado en el Códice de Dresde (límite del error $+/-4$ días).

TABLA II: Número de las revoluciones sinódicas de Venus

\begin{tabular}{lccccccccc}
\hline 4-ollin & 0 & 61 & 118 & 122 & 179 & 183 & 240 & 244 & 301 \\
4-ehecat & 1 & 62 & 119 & 123 & 180 & 184 & 241 & 245 & 302 \\
4-quiahhuitl & 11 & 72 & 129 & 133 & 190 & 194 & 251 & 255 & 312 \\
4-atl & 13 & 74 & 131 & 135 & 192 & 196 & 253 & 257 & 314 \\
4-ocelotl & 14 & 75 & 132 & 136 & 193 & 197 & 254 & 258 & 315 \\
\hline
\end{tabular}

Si 1-Tecpatl fuera tratado como una fecha venusina, indicaría $26 \mathrm{RV}=15181 \mathrm{~d}=$ $584 \mathrm{~d}$ x $26-3 \mathrm{~d}=260 \mathrm{~d}$ x $58+101 \mathrm{~d}$, y corregiría el error que conllevaba la fecha 4-Atl (13RV).

En lo que a la Luna concierne, los mínimos múltiplos del tonalpohualli que dan como efecto aproximaciones exactas de lunaciones $(\mathrm{L})$, expresadas en números enteros de días son: 5 tonalpohuallis $=44 \mathrm{~L}(+15.7 \mathrm{~h})$ y 46 tonalpohuallis $=405 \mathrm{~L}(+2,7 \mathrm{~h})$. Esta última medida que aparece en el Códice Dresde, puede ser repetida 6 veces, después habría que restar $1300 \mathrm{~d}=44 \mathrm{~L}$, y reiniciar el cómputo en el mismo nombre del día.

Los glifos con el numeral 4 en la "Piedra del Sol" permiten calcular los siguientes grupos de lunaciones menores a $405 \mathrm{~L}$ :
$11 \mathrm{~L}=325 \mathrm{~d}=260 \mathrm{~d}+65 \mathrm{~d}$
(4-Ollin $=>4$ Ehecatl $; 4$-Atl $=>4-0$ celot $=>4-$ Quiahuitl $)$
$22 \mathrm{~L}=650 \mathrm{~d}=260 \mathrm{~d} \times 2+130 \mathrm{~d}$
(4-Quiahuit $=>4-A t=>4$-Quiahuit) 


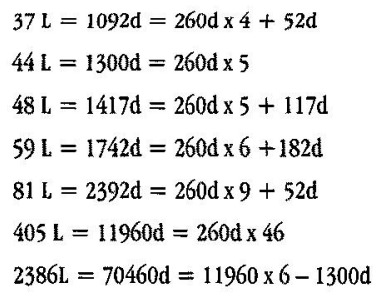

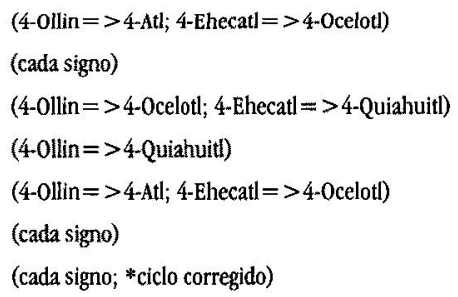

La tabla III presenta las fechas lunares que podrían calcularse a base de las inscripciones de nuestro monumento:

TABLA III: Número de las revoluciones sinódicas de la Luna

\begin{tabular}{lccccccccc}
\hline 4-ollin & 0 & 44 & $\ldots$ & $\ldots$ & 405 & 810 & 1215 & $\ldots$ & 2386 \\
4-ehecatl & 11 & 55 & $\ldots$ & $\ldots$ & 416 & 821 & 1226 & $\ldots$ & 2397 \\
4-quiahuitl & 15 & 59 & 103 & $\ldots$ & 420 & 825 & 1230 & $\ldots$ & 2401 \\
4-atl & 37 & 81 & 125 & $\ldots$ & 442 & 847 & 1252 & $\ldots$ & 2423 \\
4-ocelotl & 48 & 92 & 136 & $\ldots$ & 453 & 858 & 1263 & $\ldots$ & 2434 \\
\hline
\end{tabular}

Esta forma de cómputo permite incorporar al segundo nombre de día del monumento que quedaba sin explicación satisfactoria, es decir, a 7-0zomatli. Al hacerlo obtenemos otras medidas básicas menores del cálculo lunar.

De 4-0llin a 4-Ehecatl pueden calcularse 11 lunaciones (325 días).

De 4-0llin a 7-0zomatli pueden calcularse 12 lunaciones $(354$ días $=260 \mathrm{~d}+94 \mathrm{~d})$.

De 4-Ehecatl (11 L) a 7-0zomatli (12L) 29 días $=1 \mathrm{~L}$

De 7-0zomatli (12L) a 4-Quiahuitl (15L) $88 \mathrm{~d}=3 \mathrm{~L}$.

Los primeros 25 años de tinieblas después de la restauración del mundo actual mencionados por la Historia de los mexicanos por sus pinturas, también tienen su significado vinculado con el cómputo lunar, ya que $25 \times 365 d=9125 \mathrm{~d}=309$ lunaciones (1-Tochtli $=>13-$ Acatl). Es decir, el cómputo lunar había existido antes y podía ser reinstalado en otra edad de la Luna en 4-0llin $(+4 d)$. Podemos mencionar en este lugar un fenómeno numérico que existe entre el Sol y la Luna y que puede explicar por que en la versión del mito que nos proporciona $B$. Sahagún, la Luna destinada a ser Sol por los Dioses, intentó echarse en la hoguera cuatro veces pero no lo logró por cobardía, y de hecho saltó más tarde, en segundo turno, tan sólo en las cenizas. Pues bien, si contamos las lunas en grupos de dos, es decir cada 59 días, al cabo de un tiempo (16 ciclos) el cómputo se desajusta y falta ya un día entero para el cálculo exacto. Podríamos decir 
que la Luna se retrasa, en tanto que siguiendo el cálculo de 59 días hasta 130 ciclos, obtenemos la fecha solar de 21 años del trópico. De esta manera, el cómputo, en un principio lunar, se transforma en solar.

Es interesante observar que teniendo a 4-0llin como punto cero: 4-0celotl marca los 11 primeros años del trópico; 4-Ehecatl las 11 primeras lunaciones y 4-Quiahuitl 11 ciclos venusinos (más exacto después de 61 ciclos de Venus $=137 \times 260$ días). Esta repetición del número 11 no puede ser casual, tanto más que los cuerpos de los Xiuhcoatles, se componen de 11 cuadretes, cada uno con el símbolo de una mariposa-llama. Además, añadiendo 11 días a 7-0zomatli, que es la fecha de la décimo segunda lunación, se completa el ciclo anual básico de 365 días.

Creemos haber demostrado que el monumento Mexica llamado la "Piedra del Sol" puede proporcionar los módulos básicos para el cómputo del tiempo, señala los primeros pasos, revelando al mismo tiempo las técnicas del cálculo. Puede suponerse que la función principal de los tres nombres del día adicionales que figuran entre las astas del glifo 4-0llin, también cumplen la misma función. Fuera de lo demostrado, el papel principal de cada uno de ellos era dar nombre a definidos ciclos básicos de las revoluciones del Sol, de la Luna y de Venus: 1-Quiahuitl es la fecha del octavo año solar; 7-0zomatli designa las primeras doce lunaciones, y 1-Tecpatl, la vigésima sexta revolución de Venus.

Los datos adicionales de la Leyenda de los Soles complementan los proporcionados por el monumento, confirmando aún más la afinidad de estas dos fuentes. Según esta interpretación, los mitos nos presentan un discurso figurado de acontecimientos reales que tienen lugar en el mundo de los números y del cómputo del tiempo, es decir, en el ámbito reservado a los sabios tlamatinime.

En vista de lo dicho, el monumento con todos sus símbolos y glifos calendáricos, hace referencia tanto a la luz diurna (Sol), como a la nocturna (Venus y Luna), y por eso tal vez, sería más apropiado llamarlo Tonaltetl, es decir, "Piedra de la Luz y del Destino". 


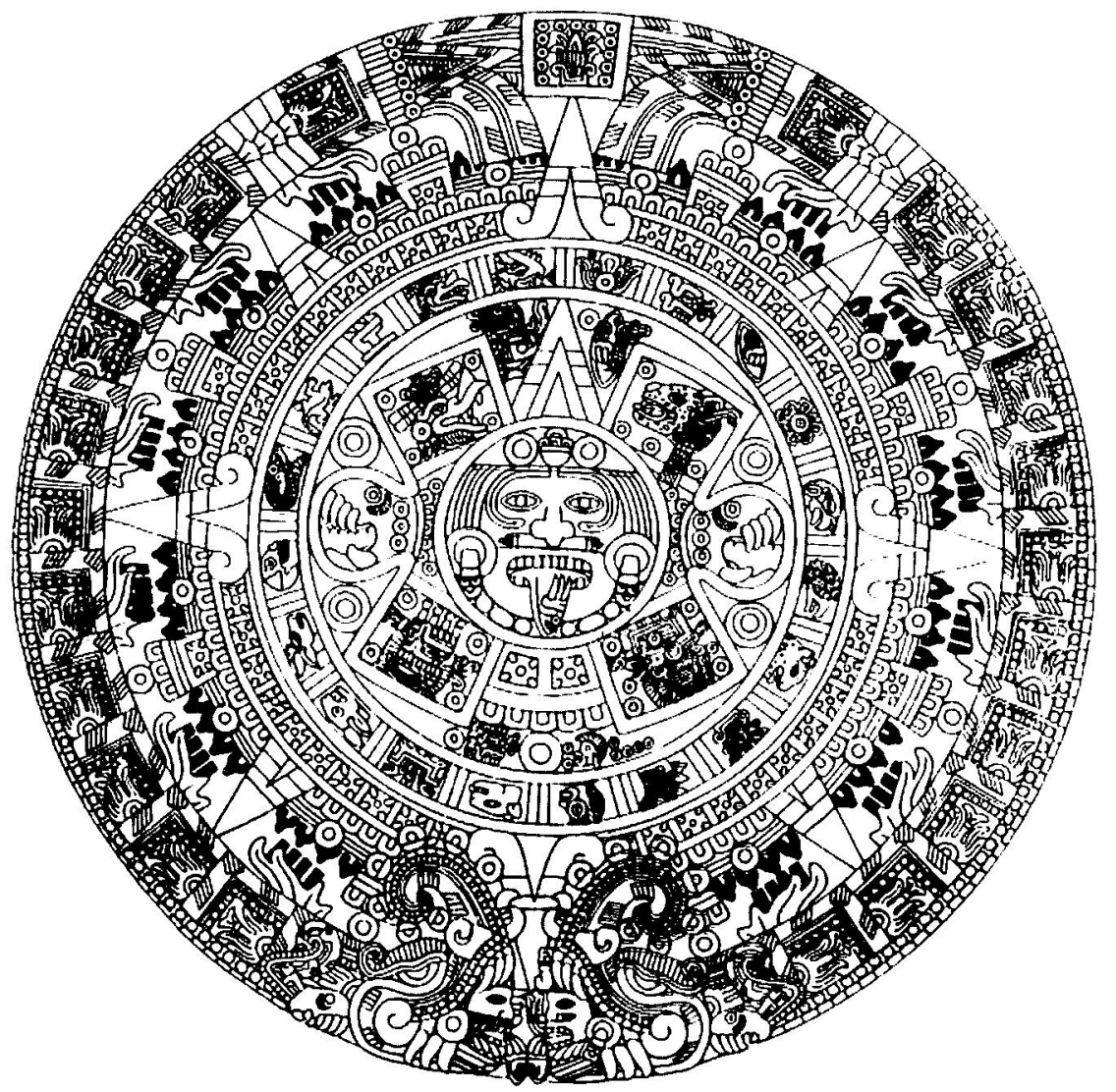

Fig. 1. La "Piedra del Sol" 


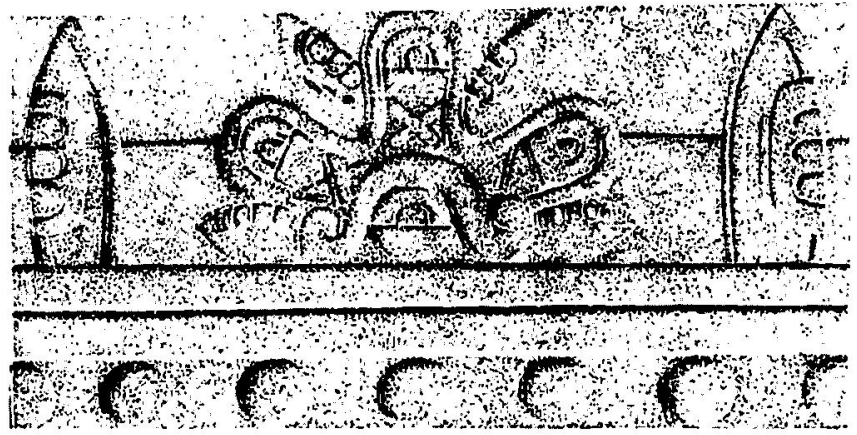

Fig. 2. Los símbolos de Venus en el canto

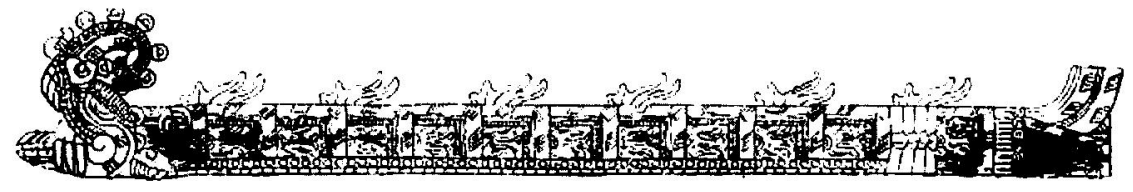

Fig. 3. El Xiuhcóatl 


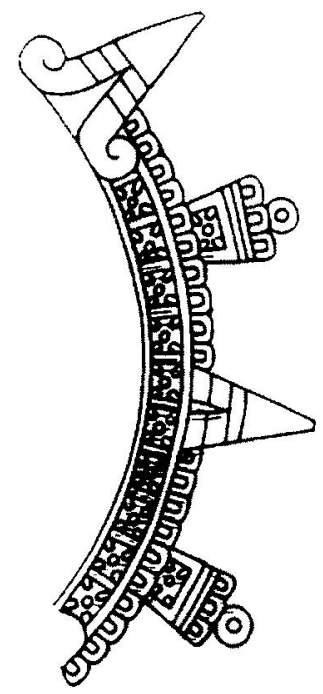

Fig. 4. Los símbolos de rayo de luz y colgajos de jade

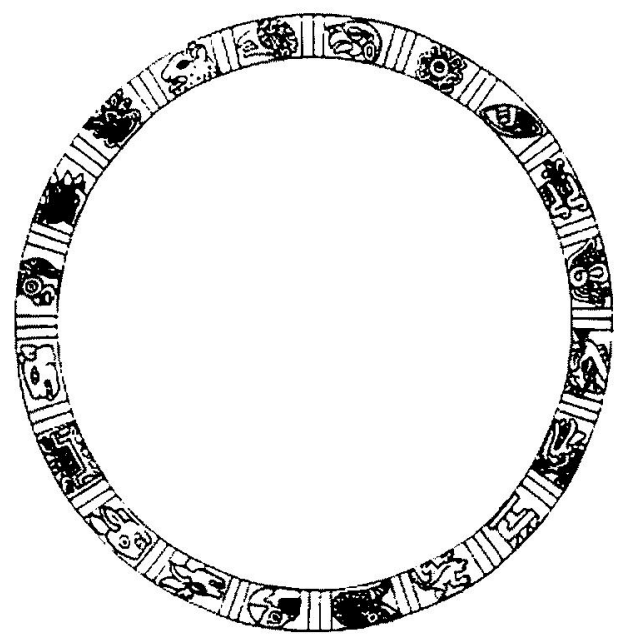

Fig. 5. Los veinte signos diurnos 

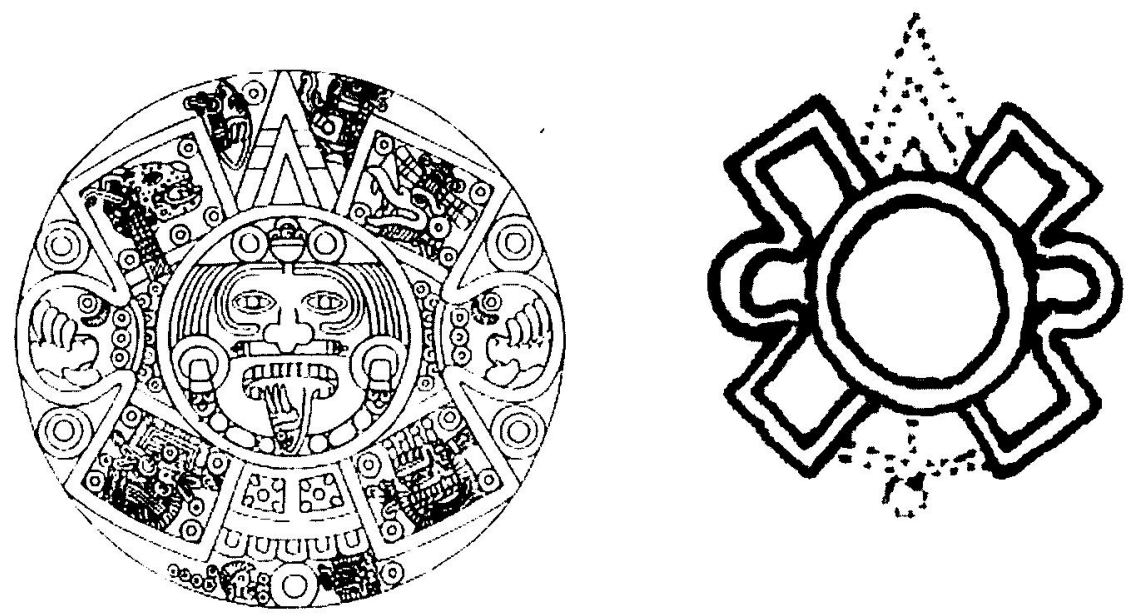

Fig. 6. El glifo 4-0llin

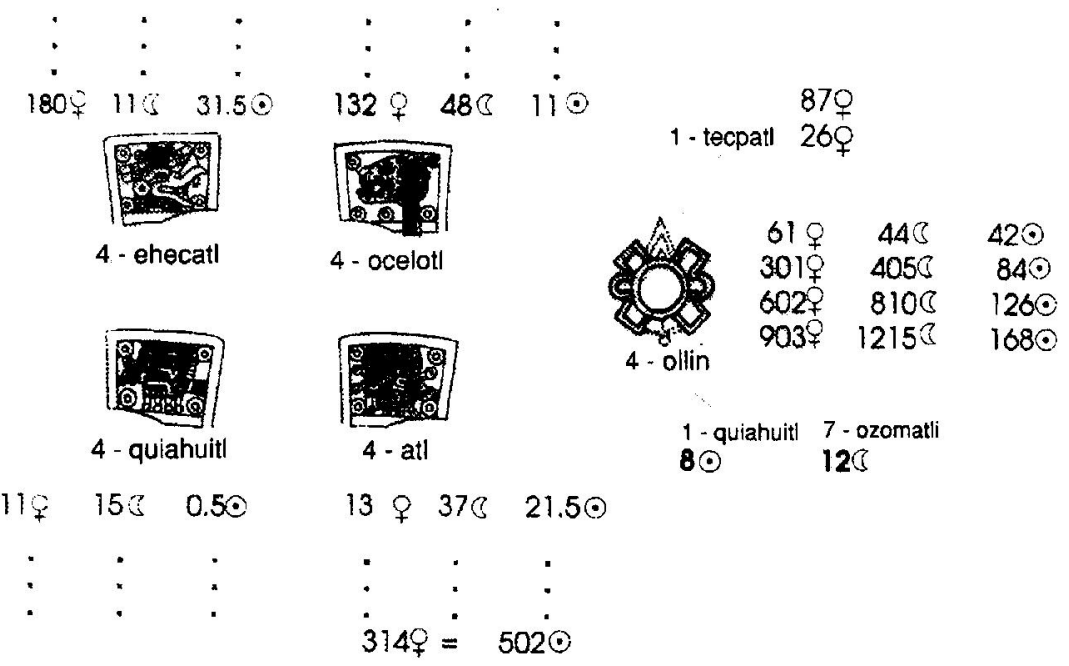

Fig. 7. Cuatro nombres del día: 4-ehecatl, 4-ocelotl, 4-quiahuitl, 4-atl 